\title{
Fat and Bone: Where are We Now?
}

\author{
Paul Dimitri ${ }^{1} \cdot$ Cliff Rosen $^{2}$
}

Received: 31 August 2016 / Accepted: 12 January 2017 / Published online: 2 February 2017

(C) Springer Science+Business Media New York 2017

For decades there has been a debate about the impact of adiposity on the skeleton and we have by no means reached a conclusion. This exciting area has moved forward considerably, in part driven by a profound increase in the prevalence of obesity and osteoporosis. Initial clinical observations and studies with DXA suggested that obesity was osteoprotective, at least in adults [1, 2]. More recently it has been suggested that obese adults are more prone to fracture which may be confined to specific skeletal regions [3]. However, the increased risk of fracture in obese children implied that the impact of obesity on bone was agedependent. This led to a number of studies examining the relationship between childhood obesity and bone with conflicting results, with some suggesting a positive relationship between adiposity and bone with others suggesting that excess fat is detrimental to the developing skeleton [2]. The issue of 'obesity' and the effect on the developing and adult skeleton was perhaps considered overly simplistic and subsequent work focusing on fat distribution demonstrated that, as with other metabolic conditions, it is visceral fat that appears to have a deleterious effect on the skeleton [4, 5] although again not all studies in adults are in agreement with this [3]. The advent of high resolution pQCT (HRpQCT) has allowed us to examine the in-vivo relationship between fat and bone at a microstructural level in children with results demonstrating that the trabecular architecture

Paul Dimitri

paul.dimitri@sch.nhs.uk

1 The Academic Unit of Child Health, Department of Paediatric Endocrinology, University of Sheffield, Sheffield, UK

2 Maine Medical Center Research Institute, Scarborough, ME 04074, Canada is more likely altered in response to obesity [6], although from cross sectional studies in adults that may not be the case [7]. Thus, the debate relating to the impact of obesity on the skeleton lives on. In this Special Edition of 'Fat and Bone', Farr, Dimitri and Walsh address the past and current research relating to the impact of obesity on bone in children and adults.

A broad range of skeletal imaging techniques have been developed to aid our understanding of skeletal mass, size and architecture and engineering models such as finite element analysis have been applied to determine bone strength. DXA remains the gold-standard clinical means of assessing skeletal parameters [8, 9], although challenges in interpreting DXA in relation to body size and proportions [10], particularly in obese children [2], and tissue thickness and distribution in $[11,12]$ have called into question the accuracy of assessing bone mass and density in relation to increasing adiposity. At the limits of in vivo scanning resolutions, HR-pQCT provides a detailed view of trabecular and cortical microarchitecture but is limited to the distal extremities and may not reflect skeletal changes elsewhere in the skeleton. In this Special Edition, the clinical considerations and diagnostic challenges in relation to skeletal imaging of children are reviewed. The challenge ahead is the development of a scanning technique that provides an accurate assessment of skeletal microarchitecture in the appendicular and axial skeleton relevant to changes in factors affecting the skeleton such as hormonal changes and physical activity in children and adults.

The discovery of hormones produced almost exclusively by adipose tissue led to a focus on the interaction between adipocytes and bone cells that may in part help to explain the clinical observations. However, results between studies can be conflicting and thus in vitro and animal in vivo findings may not always be relevant to humans. Our 
understanding of how adipokines affect and control energy regulation resulted in a shift from initial in vitro findings attempting to elucidate the direct and systemic effects of adipokines on skeletal cells, to determine how key areas of the brain such as the hypothalamus may control the skeleton. Starting with leptin at the turn of the century [13], the number of central mediators of bone metabolism has grown significantly and their roles now extended to controlling bone homeostasis rather than simply exerting a positive or negative effect on bone. Our understanding of neuronal control has extended from the sympathetic nervous system [14] to the parasympathetic and sensory nervous systems $[15,16]$. Abnormalities in the central nervous system such as vestibular dysfunction may also indirectly affect bone mass [17]. The role of the central nervous system in bone metabolism and the specific role of adiponectin in skeletal metabolism are addressed in this issue. Moving towards the skeletal microenvironment, metabolically active marrow adipose tissue has the potential to influence and control local processes [18] and may also contribute to the development of disease such as myeloma [19]. In this issue, McDonald et al. provide a comprehensive review on how cells in the bone microenvironment including adipocytes contribute to the origin of multiple myeloma, and the subsequent disease progression and drug resistance. They proposed several potential therapeutic approaches to prevent tumour progression whilst inducing bone regeneration. The paper by Craft and Scheller supports the wider view that fat cells and depots in the marrow tissue microenvironment have the potential to control multiple aspects of the skeletal metabolism, albeit that marrow adipose and white adipose tissues appear to have distinct phenotypes and evolutionary origins.

The ever-intriguing, complex and rapidly emerging relationships between fat and bone from a cellular to clinical level inspired us to bring together experts in the field to provide a series of commissioned reviews summarizing the latest advances. One of the greatest challenges is the translation of current knowledge into relevant clinical applications. Importantly, this special 'fat and bone' edition of Calcified Tissue International highlights areas that require further work to enhance our understanding of fat-bone relationships on a background of many other factors that influence skeletal metabolism.

\section{References}

1. Reid I (2010) Fat and bone. Arch Biochem Biophys 503(1):20-27
2. Dimitri P, Bishop N, Walsh JS, Eastell R (2012) Obesity is a risk factor for fracture in children but is protective against fracture in adults: a paradox. Bone 50:457-466

3. Gonnelli S, Caffarelli C, Nuti R (2014) Obesity and fracture risk. Clin Cases Miner Bone Metab 11(1):9-14

4. Gilsanz V, Chalfant J, Mo AO, Lee DC, Dorey FJ, Mittelman SD (2009) Reciprocal relations of subcutaneous and visceral fat to bone structure and strength. J Clin Endocrinol Metab 94:3387-3393

5. Fu X, Ma X, Lu H et al (2011) Associations of fat mass and fat distribution with bone mineral density in pre- and postmenopausal Chinese women. Osteoporos Int 22(1):113-119

6. Dimitri P, Jacques RM, Paggiosi M, King D, Walsh J, Taylor ZA, Frangi AF, Bishop N, Eastell R (2015) Leptin may play a role in bone microstructural alterations in obese children. $\mathrm{J}$ Clin Endocrinol Metab 100:594-602

7. Evans AL, Paggiosi MA, Eastell R, Walsh JS (2015) Bone density, microstructure and strength in obese and normal weight men and women in younger and older adulthood. J Bone Miner Res 30(5):920-928

8. Gordon CM, Bachrach LK, Carpenter TO et al (2008) Dual energy X-ray absorptiometry interpretation and reporting in children and adolescents: the 2007 ISCD pediatric official positions. J Clin Densitom 11:43-58

9. Lewiecki EM et al (2016) Best practices for dual-energy X-ray absorptiometry measurement and reporting: international society for clinical densitometry guidance. J Clin Densitom 19(2): 127-140

10. Fewtrell MS (2003) Bone densitometry in children assessed by dual X ray absorptiometry: uses and pitfalls. Arch Dis Child 88:795-798

11. Svendsen OL, Hassager C, Skodt V, Christiansen C (1995) Impact of soft tissue on in vivo accuracy of bone mineral measurements in the spine, hip, and forearm: a human cadaver study. J Bone Miner Res 10:868-873

12. LaForgia J, Dollman J, Dale MJ, Withers RT, Hill AM (2009) Validation of DXA body composition estimates in obese men and women. Obesity 17:821-826

13. Ducy $P$ et al (2000) Leptin inhibits bone formation through a hypothalamic relay: a central control of bone mass. Cell 100(2):197-207

14. Takeda $\mathrm{S}$ et al (2002) Leptin regulates bone formation via the sympathetic nervous system. Cell 111(3):305-317

15. Bajayo A et al (2012) Skeletal parasympathetic innervation communicates central IL-1 signals regulating bone mass accrual. Proc Natl Acad Sci USA 109(38):15455-15460

16. Offley SC et al (2005) Capsaicin-sensitive sensory neurons contribute to the maintenance trabecular bone integrity. J Bone Miner Res 20(2):257-267

17. Vignaux G, Besnard S, Denise P, Elefteriou F (2015) The vestibular system: a newly identified regulator of bone homeostasis acting through the sympathetic nervous system. Curr Osteoporos Rep 13(4):198-205

18. Scheller EL, Rosen CJ (2014) What's the matter with MAT? Marrow adipose tissue, metabolism, and skeletal health. Ann N Y Acad Sci 1311:14-30

19. Flank C, Fairfield H, Reagan M (2016) Signaling interplay between bone marrow adipose tissue and multiple myeloma cells. Front Endocrinol (Lausanne) 7:67 